\title{
ATRIBUIÇÃO DE CAUSALIDADE NO ENSINO SUPERIOR: ANÁLISE DA PRODUÇÃO CIENTÍFICA ${ }^{1}$
}

\author{
Danielle Ribeiro Ganda \\ Psicóloga pela Universidade Federal de Uberlândia. Mestre em Educação na área de concentração \\ Psicologia Educacional pela Universidade Estadual de Campinas (UNICAMP).
}

\begin{abstract}
Evely Boruchovitch
PhD em Educação pela University of Southern California, professora associada e livre-docente da Faculdade de Educação da Universidade Estadual de Campinas (UNICAMP). Bolsista de Produtividade do CNPq.
\end{abstract}

\begin{abstract}
Resumo
O presente artigo tem como objetivo apresentar uma análise da produção científica acerca das atribuições de causalidade de estudantes de ensino superior, com ênfase nos cursos de formação de professores. Nesse sentido, foi feito um levantamento das pesquisas envolvendo alunos universitários e professores em exercício, publicadas na literatura internacional e nacional, no período entre 1998 e 2011. Os resultados mostraram que há um maior número de trabalhos realizados internacionalmente. Constatou-se também que há poucos estudos relacionados às atribuições causais de alunos no curso de formação de professores e de docentes na ativa. De forma geral, o exame das relações entre as crenças atribuicionais e variáveis psicológicas como motivação, emoção e comportamento predominou entre as pesquisas identificadas.

Palavras-chave: crenças atribuicionais, estudantes universitários, formação de professores, revisão de literatura.
\end{abstract}

\section{ATTRIBUTION OF CAUSALITY IN HIGHER EDUCATION: ANALYSIS OF THE SCIENTIFIC PRODUCTION}

\begin{abstract}
The objective of this paper was to analyze the scientific production concerning causal attributions of higher education students. Emphasis was given to preservice teacher samples. Research reports involving university students and inservice teachers, published in national and international literature from 1998 to 2011 were examined. A greater number of research was conducted internationally. The literature regarding preservice and inservice teacher's causal attributions was scarce. Overall, the relationships between attributional beliefs and psychological variables such as motivation, emotion and behavior were the prevailing issues investigated by attributional research.
\end{abstract}

Keywords: Attributional beliefs, college students, preservice teacher's training, literature review.

\footnotetext{
${ }^{1}$ As autoras agradecem o apoio financeiro do CNPq e da CAPES.
} 


\title{
ATRIBUCIÓN DE CAUSALIDAD EN LA EDUCACIÓN SUPERIOR: ANÁLISIS DE LA PRODUCCIÓN CIENTÍFICA
}

\begin{abstract}
Resumen
Este artículo tiene como objetivo presentar un análisis de la literatura científica acerca de las atribuciones causales de los estudiantes en la educación superior, con énfasis en los cursos de formación del profesorado. En este sentido, fue un estudio de investigación con estudiantes universitarios y profesores en ejercicio, publicó la literatura internacional y nacional, entre 1998 y 2011. Los resultados mostraron que hay más trabajo por hacer a nivel internacional. También se encontró que hay pocos estudios sobre las atribuciones causales de los estudiantes en el curso de formación de maestros y profesores en servicio activo. En general, el examen de las relaciones entre las creencias atribucionales y las variables psicológicas como la motivación, la emoción y la conducta predominante entre los artículos identificados.

Palabras clave: creencias atribucionales, estudiantes universitarios, formación del profesorado, revisión de la literatura.
\end{abstract}

\section{INTRODUÇÃO}

A atribuição de causalidade refere-se ao ato de atribuir causas às situações que ocorrem consigo próprio e com os outros. Esse comportamento é concebido como algo inerente ao ser humano, uma vez que as pessoas têm necessidade de saber como ocorrem os eventos em sua vida, de tal modo que possam prever, controlar e alterar o seu destino (Heider, 1958; Weiner, 1985). Dessa forma, se uma pessoa não é aprovada numa seleção de emprego e acredita que isso ocorreu por não ter os pré-requisitos necessários da vaga, é provável que, numa próxima oportunidade, ela se prepare melhor para conseguir o êxito desejado.

Os primeiros estudos que fundamentaram a Teoria da Atribuição de Causalidade datam da década de 40, quando Fritz Heider (1944) investigou a relação entre percepção social e comportamento. Segundo o teórico a percepção que se tem sobre um dado evento é singular a cada indivíduo e ela exerce mais influência na conduta dessa pessoa do que a própria realidade (Heider, 1944, 1958). Partindo dessa premissa, diversas outras pesquisas vieram revelar que as crenças pessoais exercem considerável influência sobre outras variáveis psicológicas tais como a motivação, o comportamento, o senso de autoeficácia e as emoções (Boruchovitch, 1994; Boruchovitch \& Martini, 1997; Ferla, Valcke \& Schuyten, 2009, Graham \& Weiner, 1996; Martini \& Del Prette, 2005; Stipek \& DeCotis, 1988; Weiner, 2004).

Diante das inúmeras explicações causais que podem ser dadas pelas pessoas frente uma situação, Weiner (1979) propôs uma classificação atribuicional que inclui três dimensões: a locus de causalidade, a estabilidade e a 
controlabilidade. Na primeira, também denominada de internalidade, as causas são classificadas como internas, quando se referem às características do indivíduo (ex. esforço) ou externas, se não remetem a ele (ex. dificuldade da atividade). Na estabilidade, os fatores responsáveis pelo evento são considerados estáveis quando se mantém ao longo do tempo (ex. capacidade) e instáveis quando se alteram (ex. humor). Na controlabilidade, as causas são qualificadas como controláveis (ex. dedicação) ou incontroláveis (ex. sorte) pela pessoa.

Durante sua pesquisa houve um momento em que Weiner (1985) considerou a existência de uma quarta dimensão: a generalidade. Nesse caso, os fatores são classificados como gerais, quando abrangem diversos contextos, ou específicos, se restritos a determinada situação. Como por exemplo, um aluno poderia dizer que a inteligência é a causa de seu bom desempenho na disciplina de matemática (específico), ou afirmar que ser inteligente é uma característica que determina seu sucesso em várias outras áreas (geral). Devido às similaridades conceituais entre as dimensões "estabilidade" e "generalidade", Weiner (1988) optou por manter apenas a primeira em sua taxonomia. Entretanto, podem ser encontrados na literatura internacional, estudos recentes que adotam a generalidade (e não a estabilidade) como a terceira dimensão causal (Haugen, Lund \& Ommundsen, 2008; Roesch, Vaughn, Aldridge \& Villodas, 2009).

As investigações sobre a atribuição têm revelado que, diante de um evento, as causas mais frequentemente citadas pelas pessoas são: esforço, capacidade, dificuldade da tarefa e sorte (Boruchovitch, 1994; Boruchovitch \& Martini, 1997; Graham \& Weiner, 1996; Martini \& Boruchovitch, 2004; Weiner, 2004). Na área educacional há também outros fatores que são associados ao desempenho como o humor, a enfermidade, a fadiga e a influência de outras pessoas (Weiner, 1974). Atualmente, consideram-se ainda o "papel do professor" e o "uso de estratégias de aprendizagem" como duas importantes causas do sucesso e fracasso acadêmico dos alunos. Deve-se ressaltar que o termo "sucesso" e "fracasso" são compreendidos como qualquer atividade escolar importante para o discente na qual ele tenha (ou não) obtido êxito. Nesse caso, pode-se incluir, por exemplo, o resultado de uma prova, o rendimento em uma disciplina, a aprovação no curso, entre outros.

No contexto escolar pode-se observar a influência exercida pelas atribuições causais nas emoções, na motivação, nas expectativas e no desempenho dos 
estudantes (Boruchovitch \& Martini, 1997; Martini \& Del Prette, 2005; Stipek \& DeCotis, 1988; Weiner, 2004). O sucesso acadêmico associado a causas internas (ex. capacidade, esforço) gera sentimentos de felicidade, orgulho e satisfação. Já o fracasso pelos mesmos motivos (ex. falta de capacidade, pouco esforço) elicia emoções de culpa, vergonha e incompetência. De acordo com Weiner (1979), o êxito ou o fracasso dos alunos decorrente de causas estáveis (ex. inteligência) cria expectativas de resultados semelhantes no futuro. Logo, um fracasso causado por fatores instáveis (ex. falta de esforço) reforça a crença de que o estudante poderá obter sucesso se mudar sua conduta (ex. dedicar-se aos estudos). Quando, por exemplo, um aluno tira uma boa nota na prova e acredita que isso ocorreu devido ao seu estudo e atenção às aulas, ele normalmente se sente feliz e/ou orgulhoso e fica motivado a continuar se empenhando. Caso fracasse e perceba que não aplicou o devido esforço, ele pode ter sentimentos de culpa e/ou vergonha e intenção de melhorar seu desempenho em um próximo momento (Graham \& Weiner, 1996; Weiner, 2004).

A partir desses dados, nota-se que a atribuição de causalidade é uma importante variável a ser considerada no âmbito escolar. As crenças atribuicionais tanto dos alunos como de seus professores afetam o processo de ensino-aprendizagem, podendo comprometer o desempenho acadêmico dos discentes. Desta forma, o presente artigo tem como objetivo fazer uma análise da produção científica, internacional e nacional, referente à atribuição de causalidade de estudantes de cursos superiores, especialmente os voltados à formação de professores (Pedagogia e Licenciaturas). Nesse levantamento optou-se por incluir também os trabalhos realizados com professores em exercício (recém-formados e experientes) de tal forma a discussão sobre a temática pudesse ser ampliada. Espera-se que essa revisão de literatura trace um panorama dos avanços alcançados sobre o estudo das atribuições causais no contexto acadêmico e que contribua para a realização de futuras pesquisas nessa área.

\section{MÉTODO}

Nesse trabalho realizou-se uma consulta nas bases de dados e periódicos eletrônicos da American Psychological Association (APA), Scientific Library OnLine (SciELO), Education Resources Information Center (ERIC), Science Direct e Biblioteca Virtual em Saúde (BVS-Psi). A busca abrangeu as principais revistas 
internacionais e nacionais da área da Psicologia e da Educação, limitando-se aos estudos publicados no período entre 1998 e 2011. Para a consulta na literatura internacional foram usadas as seguintes palavras-chave: causal attributions, attributional causality, college students, teachers training course e preservice teachers. Para a busca nacional, foram usados os seguintes termos: "atribuição de causalidade", "atribuições causais", "alunos universitários", "estudantes de pedagogia" e "formação de professores".

O primeiro critério adotado para inclusão das pesquisas foi o fato de terem sido realizadas apenas com alunos universitários, excluindo aquelas com estudantes de outros níveis de ensino (fundamental e médio). Optou-se também pela escolha de trabalhos voltados a investigação da atribuição causal de professores que estejam atuando na Educação Básica. Deve-se ressaltar que só foram selecionados estudos cujo tema se referia às atribuições causais aplicadas ao contexto acadêmico e escolar, excluindo pesquisas de outras áreas como, por exemplo, a organizacional, a esportiva, entre outras.

A seleção das pesquisas foi restrita à relatos de pesquisa, não incluindo estudos teóricos e revisões de literatura. Os trabalhos são apresentados em ordem cronológica, começando pelas publicações internacionais e depois as nacionais. A descrição dos estudos é feita de forma objetiva abordando os seguintes aspectos: objetivo, autores, amostra e principais resultados. Em cada parte, são expostas inicialmente as investigações envolvendo os estudantes universitários, entre esses alguns que estão na área da Educação, e em seguida as com os professores em exercício.

\section{Pesquisas Internacionais}

Os autores Van Laar e Weiner (1998) desenvolveram um estudo com 529 estudantes do Curso de Psicologia, cujo objetivo era investigar o modo pelo qual as atribuições de causalidade para o sucesso e fracasso acadêmico, as expectativas de futuro e a autoestima exercem influência sobre a motivação dos jovens. Além disso, eles buscaram identificar o papel que a percepção de discriminação social desempenha na motivação dos alunos afro-americanos em relação a seus colegas brancos. Observou-se que os estudantes afro-americanos tendem a atribuir seu sucesso a causas internas (ex. esforço) e o fracasso a fatores externos (ex. desigualdade social). No início da faculdade esses alunos apresentam expectativas de sucesso maiores que seus colegas, mas conforme 
avançam no curso elas tendem a decrescer. Todavia, a motivação e a autoestima continuam altas.

A diferença de sexo quanto às atribuições de causalidade e a motivação de 236 universitários do Curso de Comunicação foi o tema da pesquisa realizada por Strohkirch e Hargett (1998). Na amostra total, composta por 132 mulheres e 104 homens, encontrou-se uma correlação positiva entre o nível de motivação para a aprendizagem e a estabilidade das atribuições em situações de sucesso e fracasso acadêmico. Ao compararem os sexos verificou-se que as alunas atribuíram suas realizações a fatores externos e os fracassos a causas internas com mais freqüência, do que os estudantes do sexo masculino.

Hamilton e Akter (2003) analisaram a relação entre as atribuições de causalidade e o senso de autoeficácia de 172 alunos universitários da área da Educação. Nesse estudo buscou-se validar uma escala multidimensional e multiatribucional de causalidade para o sucesso e fracasso em domínios acadêmicos e sociais. A análise dos dados indicou os alunos fazem atribuições diferenciadas conforme o contexto (ou atividade) no qual se sentem mais autoeficazes. Além disso, a escala revelou-se mais adequada para mensurar a atribuição na esfera acadêmica, não se estendendo a outras áreas da vida dos estudantes.

O propósito da pesquisa de N'gbala e Brascombe (2003) foi averiguar a influência exercida pelas atribuições nos pensamentos que se sucedem a um evento. Diante de situações fictícias, 78 alunos do Curso de Psicologia deveriam mencionar as causas que as desencadearam e quais providências precisariam ser tomadas após o ocorrido. O conteúdo e o tempo das respostas foram analisados. Os resultados mostraram que perante um fracasso causado por fatores internos e instáveis (ex. esforço), os alunos pensam mais frequentemente acerca de alternativas que pudessem ter evitado essa situação. Observou-se também que, após o fato ocorrido, o pensamento atribuicional (ex. Porque isso ocorreu?) ocorre primeiro que o de reflexão (ex. Como poderia ter sido diferente?), mas com intervalos de tempos muito próximos.

Em um estudo exploratório, realizado com 211 universitários do Curso de Psicologia da área educacional, Peterson e Schreiber (2006) investigaram as interações entre duas causas atribucionais (capacidade e esforço), as expectativas de resultado e as emoções dos alunos em trabalhos cooperativos (feitos em duplas). Ao realizarem trabalhos com colegas com alta capacidade, os 
alunos esperam obter bons resultados, independente da capacidade que se autoatribuem. Se obtiverem um mau resultado, tendem a associá-lo a causas externas (ex. dificuldade da tarefa), aumentando as expectativas de alcançar um melhor desempenho em outro momento. Situação contrária foi observada ao trabalharem com colegas percebidos como menos capazes. Quanto às emoções, os alunos relataram que o fracasso acadêmico decorrente de "pouco esforço" gera, predominantemente, "culpa" e "vergonha".

A pesquisa conduzida por Mclean, Strongman e Neha (2007) analisou a relação entre as atribuições causais, o sofrimento psíquico e o uso de estratégias de enfrentamento por 189 universitários (de vários cursos) perante uma situação de fracasso acadêmico. Constatou-se que quando o fracasso é associado a fatores internos e instáveis (ex. esforço), os alunos apresentam níveis mais baixos de sofrimento psíquico e mais freqüência nos comportamentos de enfrentamento, posto que se sentem no controle das causas do evento. Caso o insucesso seja desencadeado por motivos estáveis (ex. capacidade), os pensamentos de preocupação e os comportamentos de evitação se tornam preponderantes. Na análise da diferença entre os sexos, observou-se ainda que as mulheres demonstram maior sofrimento psíquico ao anteciparem um fracasso e que buscam mais apoio social diante de um fraco desempenho.

Cortés-Suarez e Sandiford (2008) desenvolveram uma pesquisa, com 410 alunos do Curso de Matemática, cujo objetivo foi explorar a relação entre o desempenho acadêmico e as atribuições de causalidade para o sucesso e o fracasso em uma disciplina de alta complexidade e alta taxa de repetência. Os estudantes com bom e mau rendimento na disciplina relataram que os seus resultados foram desencadeados primordialmente pelo "esforço" e em seguida pela "capacidade". Contudo, os participantes com pior desempenho mencionaram também o "grau de dificuldade da tarefa" como um importante motivo para o seu insucesso.

Os autores Haugen, Lund e Ommundsen (2008) investigaram as diferenças atribuicionais entre os padrões de personalidade de dois grupos de universitários submetidos à subculturas acadêmicas distintas. O primeiro desses foi constituído por 90 estudantes do Curso de Formação de Professores, cujo modelo de educação era voltado ao coletivismo. O segundo grupo foi composto por 60 alunos do Curso de Educação Física, cujo arquétipo de ensino era individualista. Os participantes da subcultura coletivista associaram seu sucesso a causas 
externas e o fracasso a fatores internos com mais frequência. Já o grupo dos individualistas atribuiu a si próprio os motivos tanto do seu sucesso quanto do seu fracasso.

O propósito do estudo de Ferla, Valcke e Schuyten (2009) foi averiguar as crenças atribuicionais de 448 alunos dos cursos de Psicologia e Ciência da Educação acerca de seu desempenho e a influência de seus modelos de aprendizagem em suas estratégias de estudo. Observou-se que os universitários que adotam os modelos de "aprendizagem significativa" e "memorização profunda do conteúdo", são aqueles que associam o seu sucesso acadêmico a causas controláveis (ex. esforço), possuem uma visão construtiva do conhecimento e apresentam um forte senso de autoeficácia. Eles tendem a assumir maior responsabilidade por sua aprendizagem, a acreditar em sua capacidade, a persistir por mais tempo e a fazer maior uso de estratégias de aprendizagem. Todavia, os alunos com modelos voltados à "reprodução do material" e ao "desamparo adquirido" apresentam fraco senso de autoeficácia e atribuem causas incontroláveis (ex. dificuldade da tarefa) ao seu sucesso. Além disso, eles têm uma crença de aprendizagem mecanizada, pois não se vêem capazes de compreender adequadamente o conteúdo, devendo então reproduzir a matéria da forma como lhe é ensinada pelo professor.

O trabalho realizado por Spink e Nickel (2010) visou avaliar a possível relação entre a capacidade de autorregulação de 100 universitários (de vários cursos) e as dimensões atribuicionais relacionadas a prática de exercícios físicos durante a época dos exames acadêmicos finais. Os resultados indicaram que os alunos que se sentem mais autorregulados e aqueles que têm uma causa estável para a prática de exercícios (níveis leve e médio) tendem a manter suas atividades físicas, mesmo durante os períodos de provas.

Até o momento, foram relatadas pesquisas realizadas com estudantes universitários, entre esses, alguns que estão no Curso de Formação para Professores ou em outras áreas educacionais. $\mathrm{Na}$ busca pela literatura internacional foram também encontrados alguns estudos relacionados às atribuições causais de professores em exercício que atuam na Educação Básica. Devido a sua relevância nesse contexto, esses trabalhos são descritos brevemente a seguir.

Woolfson, Grant e Campbell (2007) investigaram as diferenças das atribuições causais de 105 professores (de classes regulares e especiais) acerca 
do desempenho de alunos com dificuldade de aprendizagem. Os docentes das classes regulares demonstraram uma visão menos otimista frente à aprendizagem das crianças, relatando que o fraco rendimento dos alunos é ocasionado por fatores incontroláveis (ex. falta de capacidade). Contudo, os professores de classes especiais afirmaram que as crianças eram capazes de aprender e que eles poderiam ajudá-las a superar suas dificuldades.

Em estudo subsequente, Brady e Woolfson (2008) analisaram a relação entre as crenças atribuicionais e o senso de autoeficácia de 118 professores divididos de acordo com sua atuação, que foram: em classes regulares, auxiliares de aprendizagem ou em classes especiais. Observou-se que a autoeficácia docente exercia mais influência nas explicações causais dadas pelos participantes, do que o cargo por eles ocupado na escola. Na realidade, independentemente de estarem em classes regulares, em classes especiais ou como auxiliares, os professores com maior crença em sua capacidade de ensinar, tenderam a associar o sucesso e o fracasso de seus alunos a causas instáveis e controláveis tanto pelo discente quanto por eles próprios.

A partir desses resultados, Woolfson e Brady (2009) buscaram avaliar as possíveis relações entre a experiência profissional, a formação continuada, o senso de autoeficácia e as crenças atribuicionais de 199 professores acerca de alunos com necessidades educacionais especiais. Os dados indicaram que os docentes com menor senso de autoeficácia relatavam que o fracasso era desencadeado por causas internas do discente. Por outro lado, os profissionais mais autoeficazes disseram que os alunos não aprendiam devido à fatores externos como, por exemplo, a falta de métodos de ensino adequados às suas necessidades. Além disso, eles acreditavam que o preparo e a dedicação do professor seriam muito importantes no desenvolvimento da capacidade dos alunos. Não foram encontradas relações significativas, no que concerne ao tempo de experiência, a formação continuada e as atribuições causais dos participantes.

Em resumo, as pesquisas internacionais com estudantes universitários mostram que há relações entre as atribuições causais e a motivação, a autoestima, a autoeficácia e o desempenho acadêmico (Cortés-Suarez \& Sandiford, 2008; Ferla, Valcke \& Schuyten, 2009; Hamilton \& Akter, 2003; Mclean, Strongman \& Neha, 2007; Peterson \& Schreiber, 2006; Strohkirch \& Hargett, 1988; Van Laar \& Weiner, 1998). De modo geral, os alunos com atribuições mais apropriadas à aprendizagem (internas e controláveis) acreditam 
mais em sua capacidade, são mais motivados e apresentam um melhor rendimento. A maior parte dos estudos envolveu universitários de vários cursos como Psicologia, Comunicação, Matemática e Formação de Professores.

Os trabalhos voltados aos professores em exercício buscaram investigar a relação entre o senso de autoeficácia e as crenças atribuicionais dos docentes acerca do desempenho de seus alunos (Brady \& Woolfson, 2008; Woolfson \& Brady, 2009; Woolfson, Grant \& Campbell, 2007). Observou-se que, os professores que demonstram ser mais autoeficazes, são aqueles que acreditam no potencial do aluno em aprender e na sua própria capacidade em ensiná-los. Eles atribuem o fracasso dos estudantes a causas externas (ex. método de ensino inapropriado) e relatam ter um papel fundamental no processo de ensino/aprendizagem. Os docentes com maior senso de autoeficácia também apresentam maior clareza dos aspectos que envolvem a educação das crianças e buscam formas diferenciadas de ensino que contribuam para o desenvolvimento dos alunos.

\section{Pesquisas Nacionais}

O objetivo da pesquisa de Formiga (2004) foi comparar as atribuições de causalidade frente ao êxito acadêmico de 501 estudantes de duas universidades: uma pública e outra privada. Contatou-se que os participantes das duas instituições não se diferenciaram quanto às atribuições internas. Nesse caso, os dois grupos relataram que a capacidade e o gosto pelos estudos são as causas principais de seu sucesso acadêmico. Com relação às atribuições externas, os alunos da universidade pública afirmaram que 0 seu bom desempenho acadêmico é resultado, primordialmente, de seus procedimentos de estudo (ex. trabalhar em grupo, tirar dúvidas com o professor). Os participantes da rede privada mencionaram também as "informações anotadas em sala" como um dos fatores para o êxito. A amostra da instituição pública apresentou maior média quanto à menção do item "esforço" (ex. horas dedicadas ao estudo), enquanto que o grupo da universidade privada obteve maior freqüência no fator "autoconceito de bom estudante".

Em um estudo realizado com 181 universitários (de vários cursos), Pilati, Leão, Vieira e Fonseca (2008) analisaram, entre outros aspectos, qual a influência exercida pelas atribuições causais (dimensão controlabilidade) nas emoções e no comportamento de ajuda. Os dados revelaram que nas situações 
as quais os participantes atribuem maior controle ao colega que pede ajuda, eles tendem a culpá-lo e prestar-Ihe menos auxílio. Todavia, se acreditarem que a pessoa não tinha controle sobre o evento, esses alunos relatam sentimentos de empatia e maior tendência em ajudar.

Por meio de um questionário de autorrelato, Ganda e Boruchovitch (2010) buscaram identificar as atribuições causais e as emoções associadas ao sucesso e ao fracasso acadêmico de 27 estudantes do Curso de Pedagogia. A análise das questões demonstrou que a maioria dos participantes afirmou que a causa primordial do seu bom desempenho na faculdade é o esforço e o fator responsável pelo mau rendimento é a desmotivação. Os alunos também disseram que o seu sucesso acadêmico suscita sentimentos de satisfação e o fracasso gera frustração e culpa.

A partir desses dados, Ganda (2011) realizou uma pesquisa mais abrangente cujo tema foi a atribuição de causalidade e o uso de estratégias autoprejudiciais por 164 alunos do Curso de Pedagogia. Observou-se que o fator mais frequentemente citado como responsável pelo sucesso acadêmico foi o esforço e os sentimentos associados foram satisfação e felicidade. Quanto ao fracasso, as causas mais mencionadas pelos participantes foram a desmotivação e a falta de esforço e as emoções geradas foram frustração, culpa e tristeza. Na relação entre as duas variáveis, os alunos que relataram maior uso de estratégias autoprejudiciais (ex. procrastinação, falta de disciplina) afirmaram que são responsáveis pelo seu fracasso (ex. causas controláveis) e que o sucesso ocorre, muitas vezes, por fatores externos a eles.

Além dos artigos publicados recentemente sobre as atribuições causais de alunos universitários, foram encontradas, na literatura nacional, algumas pesquisas envolvendo professores em exercício. A primeira dessas foi desenvolvida por Neves (2002) que buscou, entre outros aspectos, analisar as possíveis relações entre as atribuições causais e as expectativas de seis professores do Ensino Fundamental sobre o desempenho de seus alunos. Observou-se que os estudantes cujos professores demonstraram percepções e expectativas favoráveis ao seu sucesso, obtiveram melhor desempenho acadêmico.

As crenças atribuicionais de 30 professoras do Ensino Fundamental acerca do desempenho acadêmico de seus alunos foram examinadas por Martini e Del Prette (2002). As participantes do estudo disseram que o docente tem um papel 
fundamental na aprendizagem das crianças. No entanto, elas tenderam a se eximir da responsabilidade nas situações em que os alunos apresentam baixo rendimento. De forma geral, as professoras relataram que o sucesso e o fracasso dos estudantes se devem às características próprias dos discentes (ex. esforço e capacidade).

Martini e Del Prette (2009) analisaram também as crenças atribuicionais relacionadas à aprendizagem dos alunos e ação docente em sala, de 33 professoras, identificadas como "facilitadoras" ou "dificultadoras" do aprendizado. $\mathrm{Na}$ comparação entre os dois grupos notou-se que as docentes "facilitadoras" mostravam maior concordância em afirmar que as relações interpessoais, o desenvolvimento socioemocional e a aprendizagem dos alunos estão intimamente relacionados. Elas também apresentaram um maior número de explicações causais ao fraco desempenho dos estudantes. Os dados sugerem que essas professoras conseguiam analisar e discriminar mais acuradamente, os componentes do processo de desenvolvimento e aprendizagem dos alunos.

Em síntese, os estudos nacionais têm se concentrado na identificação das atribuições causais e das emoções de estudantes universitários (Formiga, 2004; Ganda, 2011; Ganda \& Boruchovitch, 2010; Pilati, Leão, Vieira \& Fonseca, 2008). Os alunos relatam que o sucesso acadêmico ocorre primordialmente devido ao esforço e à capacidade. Já o fracasso ocorre principalmente pela desmotivação. Quanto aos sentimentos, o êxito tende a gerar satisfação e felicidade, enquanto o fracasso suscita frustração, culpa e tristeza. Nas pesquisas realizadas com professores em exercício observou-se que as crenças atribuicionais possuem relação com as expectativas de sucesso e fracasso e o desempenho dos alunos (Martini \& DelPrette, 2002; Martini \& Del Prette, 2009; Neves, 2002).

De forma geral, os trabalhos com alunos universitários, realizados no Brasil e no exterior, tiveram como objetivo principal investigar as relações entre as atribuições causais e os sentimentos, as expectativas de futuro, o comportamento e a motivação dos alunos (Ganda \& Boruchovitch, 2010; Haugen, Lund \& Ommundsen, 2008; Peterson \& Schreiber, 2006; Pilati, Leão, Vieira \& Fonseca, 2008; Van Laar \& Weiner, 1998). Observou-se também que a maior parte desses estudos buscou analisar as causas mencionadas pelos participantes dentro do modelo de dimensões atribuicionais, no qual a internalidade e a controlabilidade foram as mais investigadas nos dois contextos. 
No que concernem as pesquisas com professores em exercício, constatou-se uma maior preocupação em se explorar o impacto exercido pelas crenças atribuicionais na ação docente em sala de aula e no rendimento dos alunos (Neves, 2002; Martini \& Del Prette, 2009; Woolfson, Grant \& Campbell, 2007). Todavia, a relação entre as atribuições causais dos profissionais e o senso de autoeficácia para a docência foi também foco de investigação (Brady \& Woolfson, 2008; Woolfson \& Brady, 2009). Assim como nos trabalhos com universitários, as explicações causais citadas pelos professores foram classificadas segundo as dimensões da atribuição, especialmente, a controlabilidade.

\section{CONSIDERAÇÕES FINAIS}

O presente trabalho buscou fazer um levantamento das pesquisas realizadas nos últimos anos sobre a variável atribuição de causalidade no ensino superior. Especificadamente, foi feita uma revisão das publicações cuja amostra fosse de alunos universitários, em especial aqueles inseridos nos cursos de formação de professores e áreas afins, e de professores em exercício. Na análise inicial pôde-se observar que a literatura internacional contém um maior número de artigos quando comparados às publicações nacionais. Contudo, nos últimos anos, tem-se observado um pequeno aumento na quantidade de pesquisas divulgadas nacionalmente.

Apesar da relevância do tema, corroborada pelos resultados das pesquisas ora apresentados, notou-se uma produção escassa sobre a atribuição de causalidade com alunos em processo de formação para docência e de docentes na ativa, tanto a nível internacional como nacional. Dessa forma, tendo em vista a importância das crenças atribuicionais na compreensão do processo de ensino e aprendizagem, recomenda-se que futuros trabalhos sejam empreendidos, avaliando as atribuições dos alunos na universidade e também durante sua atuação profissional.

Em linhas gerais, acredita-se que os objetivos propostos nesse artigo foram alcançados e permitiram visualizar um panorama das pesquisas recentes sobre atribuição de causalidade desenvolvidas com alunos do ensino superior. Deve-se ressaltar, entretanto, que essa revisão de literatura não incluiu livros, capítulos de livros e teses que possam ter investigado a temática. Assim sendo, é possível que a produção científica sobre o tema seja consideravelmente superior à mapeada no presente estudo. Sugere-se que novos trabalhos abordem também 
essas publicações, ampliando a investigação sobre as atribuições causais em contexto educacional.

\section{REFERÊNCIAS}

Boruchovitch, E. (1994). As variáveis psicológicas e o processo de aprendizagem: uma contribuição para a Psicologia Escolar. Psicologia: Teoria e Pesquisa, 10(1), 111-128.

Boruchovitch, E. \& Martini, M. L. (1997). As atribuições de causalidade para o sucesso e o fracasso escolar e a motivação para a aprendizagem de crianças brasileiras. Arquivos Brasileiros de Psicologia, 49(3), 59-71.

Brady, K., \& Woolfson, L. (2008). What factors influence teacher attributions for children's difficulties in learning? British Journal of Educational Psychology, 78(4), 115-121.

Cortés-Suárez, G. \& Sandiford, J. R. (2008). Causal attributions for success or failure of students in college algebra. Community College Journal of Research and Practice, 32, 325-346.

Ferla, J.; Valcke, M.; Schuyten, G. (2009). Student models of learning and their impact on study strategies. Studies in Higher Education, 34(2),185-202.

Formiga, N. (2004). Atribuição de causalidade e rendimento acadêmico: predição e diferença em alunos da universidade pública e privada. Revista de Psicologia da UNC, 2(1), 3-14.

Ganda, D. R. (2011). Atribuições de Causalidade e Estratégias Autoprejudiciais de Alunos de Curso de Formação de Professores. Dissertação de Mestrado. Faculdade de Educação. Universidade Estadual de Campinas, Campinas. São Paulo, Brasil.

Ganda, D. R. \& Boruchovitch, E. (2010). As Atribuições de Causalidade para o Sucesso e Fracasso acadêmico de Alunos de um Curso de Pedagogia. Anais do VII Encontro de Pesquisa em Educação da Região Sul - ANPED. Londrina, PR.

Graham, S. \& Weiner, B. (1996). Theories e Principles of motivation. In: D. C. Berliner \& R. C. Calfee (Orgs.), Handbook of Educational Psychology (pp. 63-84). New York: Simon \& Schuster Macmillan.

Hamilton, R. J. \& Akhter S. (2003). Psychometric Properties of the Multidimensional-Multiattributional Causality Scale. Educational and Psychological Measurement, 62(5), 802-817. 
Haugen, R., Lund, T., Ommundsen, Y. (2008). Personality Dispositions,

Expectancy and Context in Attributional Thinking. Scandinavian Journal of Educational Research, 52(2), 171-185.

Heider, F. (1944). Social perception and phenomenal causality. Psychological Review, 51, 358-374.

Heider, F. (1958). The Psychology of interpersonal relations. New York: Wiley.

Martini, M. L. \& Boruchovitch, E. (2004). A teoria da atribuição de causalidade: contribuições para a formação e atuação de educadores. Campinas: Alínea.

Martini, M. L., Del Prette, Z. A. P. (2002) Atribuições de Causalidade para o sucesso e fracasso escolar dos seus alunos por professores do ensino fundamental. Interação em Psicologia, 6(2), 149-156.

Martini, M. L. \& Del Prette, Z. (2005). Atribuições de causalidade e afetividade de alunos de alto e baixo desempenho acadêmico em situações de sucesso e fracasso escolar. Revista Interamericana de Psicologia, 39(3), 355-368.

Martini, M. \& DelPrette, Z. (2009). Crenças docentes e implicações para o processo de ensino-aprendizagem. Revista Semestral da Associação Brasileira de Psicologia Escolar e Educacional, 13, 75-85.

Mclean, J. A., Strongman, K. T. \& Neha, T. N. (2007) Psychological Distress, Causal Attributions, and Coping. New Zealand Journal of Psychology, 36(2), 85-94.

Neves, L. F. (2002). Um estudo sobre as relações entre a percepção e as expectativas dos professores e dos alunos e o desempenho em matemática. Dissertação de Mestrado. Universidade Estadual de Campinas: Campinas, São Paulo.

N'gbala, A. \& Branscombe, N. R. (2003). Causal attribution and counterfactual thinking - when does performing one facilitate performance of the other. Swiss Journal of Psychology, 62(4), 2003, 209-218.

Nickel, D. \& Spink, K. S. (2010). Attributions and self-regulatory efficacy for health-related physical activity. Journal of Health Psychology, 15(1), 53-63.

Peterson, S. \& Schreiber, J. B. (2006). An Attributional Analysis of Personal and Interpersonal Motivation for Collaborative Projects. Journal of Educational Psychology, 98(4), 777-787.

Pilati, R.; Leão, M., Vieira, J. \& Fonseca, M (2008). Efeitos da atribuição de causalidade e custo pessoal sobre a intenção de ajuda. Estudos de Psicologia 2008, 13(3), 213-221. 
Roesch, S. C., Vaughn, A. A., Aldridge, A. A. \& Villodas, F. (2009). Internacional Journal of Psychology, 44(5), 393-400.

Spink, K. S. \& Nickel, D. (2010). Self-regulatory efficacy as a mediator between Attributions and Intention for Health-related Physical Activity. Journal of Health Psychology, 15(1), 75-84.

Stipek, D., \& DeCotis, K. (1988). Children's understanding of the implications of causal attributions for emotional experiences. Child Development, 59(6), 1601-10.

Strohkirch, C. \& Hargett, J. (1998). A preliminary analysis of sex differences in attributional patterns and self-esteem levels. Paper presented at the Annual Meeting of the Central States Communication Association. Chicago, Apr., 25.

Van Laar, C. \& Weiner, B. (1998). Attributions and expectancies as determinants of achievement in black students. Paper presented at the Annual Convention of the American Psychologycal Association. 106th, San Francisco, Aug, 14-18.

Weiner, B. (1974). Achievement motivation and attribution theory. Morristown, N. J.: General Learning Press.

Weiner, B. (1979). A theory of motivation for some classroom experiences. Journal of Educational Psychology, 71, 3-25.

Weiner, B. (1985). An attributional theory of achievement motivation and emotion. Psychological Rewiew, 92(4), 548-573.

Weiner, B. (1988). Attribution theory in education. Revista Portuguesa de Educação, 1(1), 21-25.

Weiner, B. (2004). Attribution theory revisited: Transforming cultural plurality into theoretical unity. In: Research on Sociocultural influences on motivation and learning. Information Age Publishing, Inc., 13-29.

Woolfson, L. M.; Brady, K. (2009). An investigation of factors impacting on mainstream teacher's beliefs about teaching students with learning difficulties. Educational Psychology, 29(2), 221-238.

Woolfson, L.; Grant, E. \& Campbell, L. (2007). A Comparison of Special, General and Support Teachers' Controllability and Stability Attributions for Children's Difficulties in Learning. Educational Psychology, 27(2), 295-306. 
Contato: danielleganda@hotmail.com, evely@unicamp.br

Recebido em: 09/02/2011

Revisado em: 29/04/2011

Aceito em: 05/05/2011 